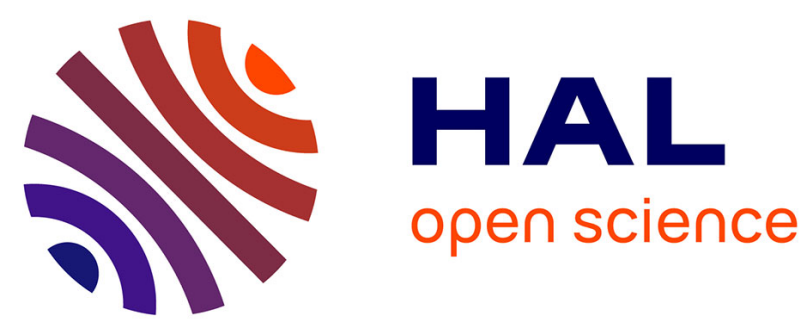

\title{
fMRI BOLD signal decomposition using a multivariate low-rank model
}

Hamza Cherkaoui, Thomas Moreau, Abderrahim Halimi, Philippe Ciuciu

\section{To cite this version:}

Hamza Cherkaoui, Thomas Moreau, Abderrahim Halimi, Philippe Ciuciu. fMRI BOLD signal decomposition using a multivariate low-rank model. Eusipco 2019 - 27th European Signal Processing Conference, Sep 2019, Corunna, Spain. hal-02163497v2

\section{HAL Id: hal-02163497 \\ https://hal.science/hal-02163497v2}

Submitted on 9 Sep 2019

HAL is a multi-disciplinary open access archive for the deposit and dissemination of scientific research documents, whether they are published or not. The documents may come from teaching and research institutions in France or abroad, or from public or private research centers.
L'archive ouverte pluridisciplinaire HAL, est destinée au dépôt et à la diffusion de documents scientifiques de niveau recherche, publiés ou non, émanant des établissements d'enseignement et de recherche français ou étrangers, des laboratoires publics ou privés. 


\title{
fMRI BOLD signal decomposition using a multivariate low-rank model
}

\author{
Hamza Cherkaoui*, Thomas Moreau ${ }^{\dagger}$, Abderrahim Halimi $^{\ddagger}$ and Philippe Ciuciu* \\ ${ }^{*}$ CEA Saclay, Univ. Paris-Saclay, 91191 Gif-sur Yvette, France. \\ ${ }^{\dagger}$ Parietal Team, INRIA Saclay, Université Paris-Saclay, Saclay, France. \\ $\ddagger$ School of Engineering and Physical Sciences, Heriot-Watt University, Edinburgh UK.
}

\begin{abstract}
Standard methodologies for functional Magnetic Resonance Imaging (fMRI) data analysis decompose the observed Blood Oxygenation Level Dependent (BOLD) signals using voxelwise linear model and perform maximum likelihood estimation to get the parameters associated with the regressors. In task fMRI, the latter are usually defined from the experimental paradigm and some confounds whereas in resting-state acquisitions, a seedvoxel time-course may be used as predictor. Nowadays, most fMRI datasets offer resting-state acquisitions, requiring multivariate approaches (e.g., PCA, ICA, etc) to extract meaningful information in a data-driven manner. Here, we propose a novel low-rank model of fMRI BOLD data but instead of considering a dimension reduction in space as in ICA, our model relies on convolutional sparse coding between the hemodynamic system and a few temporal atoms which code for the neural activity inducing signals. A rank-1 constraint is also associated with each temporal atom to spatially map its influence in the brain. Within a variational framework, the joint estimation of the neural signals and the associated spatial maps is formulated as a nonconvex optimization problem. A local minimizer is computed using an efficient alternate minimization algorithm. The proposed approach is first validated on simulations and then applied to task fMRI data for illustration purpose. Its comparison to a state-of-the-art approach suggests that our method is competitive regarding the uncovered neural fingerprints while offering a richer decomposition in time and space.
\end{abstract}

\section{INTRODUCTION}

Context. Functional magnetic resonance imaging (fMRI) non-invasively records brain activity by dynamically measuring the blood oxygenation level-dependent (BOLD) contrast. The latter reflects the local changes in the deoxyhemoglobin concentration in the brain [1] and thus indirectly measures neural activity through the neurovascular coupling. This coupling is usually characterized as a linear and time-invariant system and thus summarized by its impulse response, the so-called hemodynamic response function (HRF) [2, 3]. Its estimation links the observed BOLD signal to the underlying neural activity, which can in turn be used to understand cognitive processes in the healthy brain or to uncover functional alteration in pathological condition. Nevertheless, as a voxel contains about one hundred of thousands neurons for a typical spatial resolution $(1.5 \mathrm{~mm}$ isotropic) the underlying neural activity signals are associated with possibly different temporal fingerprints.

Related works. The classical data analysis approach proposes to decompose the BOLD signal using multiple predefined regressors. Each regressor is a time series that models the given temporal signature of an experimental stimulus or task, named condition, convolved with a canonical HRF [4]. Those timecourses are concatenated into a so-called design matrix, and fitted to the observed BOLD data. The estimated coefficients provide the encoding localization of each condition in the brain [4]. The main limitation of this massively univariate approach is twofold: first, it treats one voxel at a time using the same model; second, it requires the prior knowledge of the experimental paradigm. For these reasons, unsupervised multivariate methods have been introduced in the literature to deal with paradigm-free fMRI datasets such as restingstate recordings. The most famous are likely the principal component analysis (PCA) [5] and the independent component analysis (ICA) [6, 7]. However, all these techniques directly work on the measured BOLD time series and do not deconvolve them to highlight neural activities. An alternative consists in distangling the neurovascular coupling by deconvolving the BOLD signal using a well chosen HRF [8-10] and thus recovering voxel-wise neural activation signals. Those approaches provide as many components as the number of voxels. Those components are then used to explore the underlying structure in the data by quantifying either how they cluster together or their functional connectivity.

Goals and contributions. This paper presents a new algorithm that aims to offer a rich decomposition of the BOLD signal using low-rank sparse decomposition. Following the ideas developed in the dictionary learning literature [11, 12], our approach consists in modeling the observed BOLD signal as a linear combination of a limited number of temporal atoms whose first-order derivative is sparse. In that purpose, we introduce spatio-temporal maps which take the neurovascular coupling (temporal aspect) and the localization of activations (spatialization) into account. Then, we jointly estimate those temporal atoms and the associated maps with properly selected constraints. The resulting optimization problem is nonconvex but an approximated solution can be computed using an alternate minimization algorithm with an efficient procedure to be performed at each step.

Section II introduces our modeling of the BOLD signal and presents our estimation algorithm. Next, our technique is evaluated against state-of-the-art algorithm in Section III. Conclusions and future work are discussed in Section IV. 


\section{LOW RANK DECOMPOSITION OF THE BOLD SIGNAL}

In this section, we present our modeling of the BOLD signal and derive an efficient algorithm to estimate its parameters.

Notation. $\quad x_{i}$ denotes the $\mathrm{i}^{\text {th }}$ entry in vector $\boldsymbol{x}$. Let $\widetilde{T}=$ $T-L+1$, the convolution of two signals $\boldsymbol{z} \in \mathbb{R}^{1 \times \widetilde{T}}$ and $\boldsymbol{d} \in \mathbb{R}^{1 \times L}$ is denoted by $\boldsymbol{z} * \boldsymbol{d} \in \mathbb{R}^{1 \times T}$. For $\boldsymbol{D} \in \mathbb{R}^{P \times L}$, $\boldsymbol{z} \dot{*} \boldsymbol{D} \in \mathbb{R}^{P \times T}$ is obtained by convolving each row of $\boldsymbol{D}$ with $\boldsymbol{z}$. We denote $\boldsymbol{L}$ the discrete integration operator such that $\forall \boldsymbol{z} \in \mathbb{R}^{1 \times \widetilde{T}}, \quad \boldsymbol{L} \boldsymbol{z}=\left(\sum_{j=1}^{i} z_{j}\right)_{i \in\{1 . . \widetilde{T}\}}$.

\section{A. Linear and time-invariant modeling}

A common model for the multivariate ( $P$ voxels, $T$ scans $)$ BOLD data $\boldsymbol{X} \in \mathbb{R}^{P \times T}$ with $\boldsymbol{X}=\left(\boldsymbol{x}_{j}\right)_{j \in\{1 . . P\}}$ is the linear and time-invariant model (LTI) [3], where for each voxel, the measured time series, denoted $\boldsymbol{x}_{j} \in \mathbb{R}^{1 \times T}$, is the convolution of a neural activation signal, denoted $\widetilde{\boldsymbol{a}}_{j} \in \mathbb{R}^{1 \times \widetilde{T}}$ with a given HRF, here denoted $\boldsymbol{v} \in \mathbb{R}^{1 \times L}$, such that $\boldsymbol{x}_{j}=\boldsymbol{v} * \widetilde{\boldsymbol{a}}_{j}+$ $\boldsymbol{e}_{j}$ where $\boldsymbol{e}_{j} \in \mathbb{R}^{1 \times T}$ refers to an additive white Gaussian noise [13]. Typically, the HRF $\boldsymbol{v}$ has a restricted support in time and quantifies the neurovascular coupling in a specific region of the brain. For the sake of simplicity, the same HRF shape is usually considered for the whole brain and we choose the canonical SPMs double gamma function HRF, as mention in [14]. This model extends as follows:

$$
\boldsymbol{X}=\boldsymbol{v} \dot{\boldsymbol{A}}+\boldsymbol{E}
$$

with $\boldsymbol{E}=\left(\boldsymbol{e}_{j}\right)_{j \in\{1 . . P\}} \in \mathbb{R}^{P \times T}$ and $\widetilde{\boldsymbol{A}}=\left(\widetilde{\boldsymbol{a}}_{j}\right)_{j \in\{1 . . P\}} \in$ $\mathbb{R}^{P \times T}$. The activation signals $\widetilde{\boldsymbol{A}}$ capture, in an univariate manner, the periods of time during which some voxels are involved in task performance (or in spontaneous BOLD signal fluctuations). In this univariate model, $P$ independent neural activation signals $\left(\widetilde{\boldsymbol{a}}_{j}\right) j \in\{1 . . P\}$ are learned, one for each voxel. In our work, we propose to learn $K$ temporal activations $\left(\boldsymbol{a}_{k}\right)_{k \in\{1 . . K\}}$ and their associated spatial maps $\boldsymbol{u}_{k} \in \mathbb{R}^{P \times 1}$, as we aim to recover $K$ distinctive functional networks with a specific temporal fingerprint. This can be modeled by replacing each vector $\widetilde{\boldsymbol{a}}_{j}$ in Eq. (1) with a linear combination of the activations $\left(\boldsymbol{a}_{k}\right)_{k \in\{1 . . K\}}$. A classical assumption for these temporal activation signals is to consider them piecewise constant as in [8-10]. To that aim, we model them as $\boldsymbol{a}_{k}=\boldsymbol{L} \boldsymbol{z}_{k}$, where $\boldsymbol{z}_{k}$ is sparse. The spatial configuration $\boldsymbol{u}_{k} \in \mathbb{R}^{P \times 1}$ encodes which voxels are linked to a given temporal activation $\boldsymbol{L} \boldsymbol{z}_{k} \in \mathbb{R}^{1 \times \widetilde{T}}$. In our work, we propose a fixed HRF $\boldsymbol{v}$ and define the rank-1 spatio-temporal maps $\boldsymbol{u}_{k} \boldsymbol{v}^{\top} \in \mathbb{R}^{P \times V}$ as the convolution kernel with the neural activity, as depicted in Fig. 1. Learning the HRF will be deferred to future work. Our forward model for BOLD fMRI data thus reads:

$$
\boldsymbol{X}=\sum_{k=1}^{K}\left(\boldsymbol{L} \boldsymbol{z}_{k}\right) \dot{*}\left(\boldsymbol{u}_{k} \boldsymbol{v}^{\top}\right)+\boldsymbol{E} .
$$

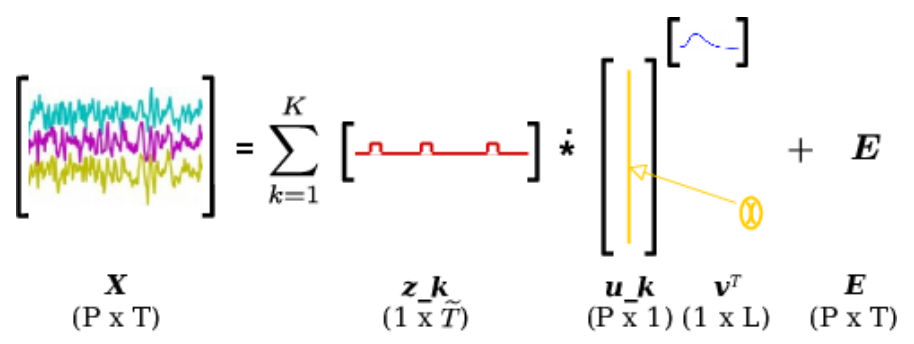

Fig. 1: Illustration of the low-rank BOLD signal model (the colors are there for illustrative purposes).

\section{B. Optimization problem}

The spatial maps $\left(\boldsymbol{u}_{k}\right)_{k \in\{1 . . K\}}$ and the neural activation signal $\left(\boldsymbol{z}_{k}\right)_{k \in\{1 . . K\}}$, from Eq. (2), can be jointly estimated by solving the following constrained minimization problem:

$$
\begin{aligned}
J\left(\left(\boldsymbol{u}_{k}\right)_{k},\left(\boldsymbol{z}_{k}\right)_{k}\right)= & \frac{1}{2}\left\|\boldsymbol{X}-\sum_{k=1}^{K}\left(\boldsymbol{L} \boldsymbol{z}_{k}\right) \dot{*}\left(\boldsymbol{u}_{k} \boldsymbol{v}^{\top}\right)\right\|_{F}^{2}+\lambda \sum_{k=1}^{K}\left\|\boldsymbol{z}_{k}\right\|_{1} \\
\text { subject to } & \left\|\boldsymbol{u}_{k}\right\|_{1}=\eta \quad \text { and } \quad u_{k j} \geq 0
\end{aligned}
$$

To be consistent with [8-10], we enforce the temporal atoms $\left(\boldsymbol{z}_{k}\right)_{k \in\{1 . . K\}}$ to be sparse in order to constrain $\left(\boldsymbol{L} \boldsymbol{z}_{k}\right)_{k \in\{1 . . K\}}$ to be piecewise constant signals. Indeed, most of experimental paradims in fMRI propose to model task-related evoked activity using block signals. Moreover, to limit the indetermination in the convolution we impose the non-negativity in the entries of the spatial maps $\left(\boldsymbol{u}_{k}\right)_{k \in\{1 . . K\}}$. Last, to deal with the scale ambiguity - the fact that any solution pair $\left(\widehat{\boldsymbol{u}}_{k}, \widehat{\boldsymbol{z}}_{k}\right)$ is known up to a multiplicative constant - we set $\forall k \in\{1 . . K\} \quad\left\|\boldsymbol{u}_{k}\right\|_{1}=$ $\eta$, with $\eta \in \mathbb{R}_{+}$being a parameter of our method that fixes the magnitude of each spatial maps. This optimization problem is biconvex in $\left(\boldsymbol{u}_{k}\right)_{k \in\{1 . . K\}}$ and $\left(\boldsymbol{z}_{k}\right)_{k \in\{1 . . K\}}$, meaning that it is convex in each variable but not jointly convex. We minimize Eq. (3) using a block-coordinate descent algorithm, where we alternate the minimization between the two convex problems in $\left(\boldsymbol{u}_{k}\right)_{k \in\{1 . . K\}}$ and in $\left(\boldsymbol{z}_{k}\right)_{k \in\{1 . . K\}}$. Algorithm 1 details these two steps.

We minimize each step with an accelerated forwardbackward algorithm [15] with Armijo backtracking line search [16]. Recall that for $\boldsymbol{v} \in \mathbb{R}^{1 \times L}, \boldsymbol{a} \in \mathbb{R}^{1 \times \widetilde{T}}$ and $\boldsymbol{x} \in \mathbb{R}^{1 \times T}$ $\nabla_{\boldsymbol{z}}\left(\frac{1}{2}\|\boldsymbol{x}-\boldsymbol{v} * \boldsymbol{a}\|_{2}^{2}\right)=-\boldsymbol{v} *(\boldsymbol{x}-\boldsymbol{v} * \boldsymbol{a})$ with the time flipped HRF $v_{j}^{\dagger}=v_{\widetilde{T}-j}$, thus our gradient steps read:

$$
\begin{aligned}
& \nabla_{\boldsymbol{z}_{\ell}} F_{\boldsymbol{u}_{k}}\left(\boldsymbol{z}_{k}\right)=-\boldsymbol{L}^{\top}\left(\left(\boldsymbol{u}_{\ell} \boldsymbol{v}^{\top}\right)^{\dagger} \dot{*}\left(\boldsymbol{X}-\sum_{k=1}^{K}\left(\boldsymbol{L} \boldsymbol{z}_{k}\right) \dot{*}\left(\boldsymbol{u}_{k} \boldsymbol{v}^{\top}\right)\right)\right), \\
& \nabla_{\boldsymbol{u}_{\ell}} F_{\boldsymbol{z}_{k}}\left(\boldsymbol{u}_{\boldsymbol{k}}\right)=-\boldsymbol{v}\left(\left(\boldsymbol{L} \boldsymbol{z}_{\ell}\right)^{\dagger} \dot{*}\left(\boldsymbol{X}-\sum_{k=1}^{K}\left(\boldsymbol{L} \boldsymbol{z}_{k}\right) \dot{*}\left(\boldsymbol{u}_{k} \boldsymbol{v}^{\top}\right)\right)\right) .
\end{aligned}
$$

The computation of $\nabla_{\boldsymbol{z}_{\ell}} F_{\boldsymbol{u}_{k}}\left(\boldsymbol{z}_{k}\right)$ is optimized by precomputing $-\boldsymbol{L}^{\top}\left(\boldsymbol{u}_{\ell} \boldsymbol{v}^{\top}\right)^{\dagger} \dot{*} \boldsymbol{X}$ and $\boldsymbol{L}^{\top}\left(\boldsymbol{u}_{\ell} \boldsymbol{v}^{\top}\right)^{\urcorner} \dot{*}\left(\boldsymbol{u}_{k} \boldsymbol{v}^{\top}\right) \boldsymbol{L}$ while that of $\nabla_{\boldsymbol{u}_{\ell}} F_{\boldsymbol{z}_{k}}\left(\boldsymbol{u}_{k}\right)$ is accelerated by pre-computing $-\boldsymbol{v}\left(\boldsymbol{L} \boldsymbol{z}_{\ell}\right)^{\dagger} \dot{*} \boldsymbol{X}$ and $\boldsymbol{v}\left(\boldsymbol{L} \boldsymbol{z}_{\ell}\right)^{\dagger} \dot{*} \boldsymbol{v}^{\top}\left(\boldsymbol{L} \boldsymbol{z}_{k}\right)$, as those quantities remain constant during these respective steps. 


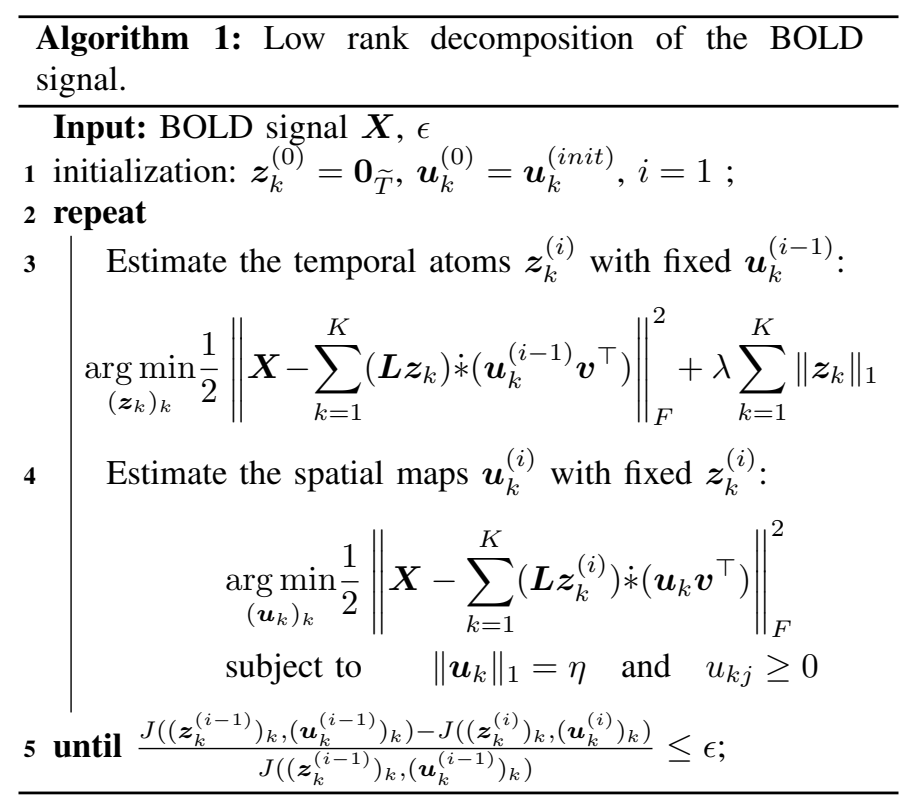

The proximal operator of $g_{z}\left(\left(\boldsymbol{z}_{k}\right)_{k}\right)=\lambda \sum_{k=1}^{K}\left\|\boldsymbol{z}_{k}\right\|_{1}$ is the soft-thresholding defined coordinate-wise as $\operatorname{sign}(z)(|z|-\lambda)_{+}$. For the constraint $g_{u}\left(\left(\boldsymbol{u}_{k}\right)_{k}\right)=I_{\left\|\boldsymbol{u}_{k}\right\|_{1}=\eta}+I_{u_{k j} \geq 0}$ on the spatial maps, the corresponding proximal operator is given

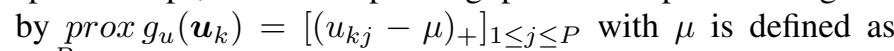
$\sum_{j=1}^{P} \max \left\{0, u_{k j}-\mu\right\}=\eta$ and an efficient implementation can be found in [17].

We early-stopped the main loop when each main iteration does not decrease sufficiently the cost function. In practice less than 50 iterations of the main loop were needed to converge.

Owing to the global non-convexity, this approach converges to a local minimizer of Eq. (3), which may be sub-optimal for our estimation objective. To initialize the spatial maps $\left(\boldsymbol{u}_{k}\right)_{k=1 \ldots K}$, we draw each entry as a centered Gaussian variable with variance 1 . To limit the impact of the initialization selection, we run multiple times the minimization.

\section{NUMERICAL EXPERIMENTS}

In this section, we validate our approach on simulation and illustrate its application to real fMRI data. All experiments were performed in Python and our implementation, as well as the scripts for experimental validation ${ }^{1}$, are freely available online.

\section{A. Results on synthetic data}

Artificial BOLD time series. We randomly generated $P=100$ BOLD signals $\boldsymbol{X}$. Each time series $\boldsymbol{x}_{j}$ was defined as the linear combination of two temporal atoms $\left(\boldsymbol{z}_{1}, \boldsymbol{z}_{2}\right)$ comprising two blocks each whose duration was fixed to $10 \mathrm{~s}$ and the magnitude was randomly drawn from a Gaussian distribution centered on 1.0. The weights are defined in two spatial maps $\left(\boldsymbol{u}_{1}, \boldsymbol{u}_{2}\right)$ with a single non-zero pixel in each map. To simulate a realistic scenario, we chose a TR of

\footnotetext{
${ }^{1}$ https://github.com/CherkaouiHamza/seven
}

(a) Temporal atoms
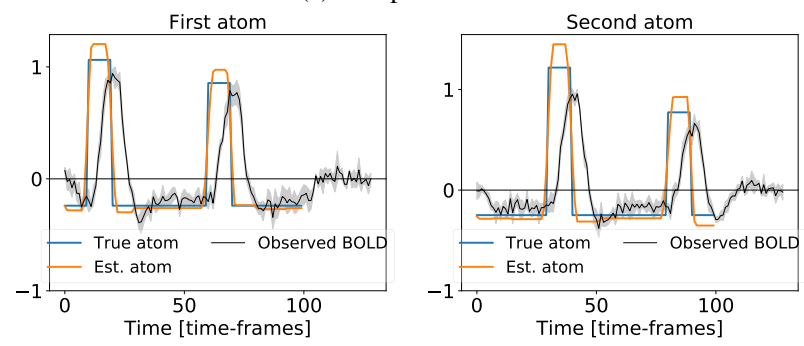

(b) Spatial maps

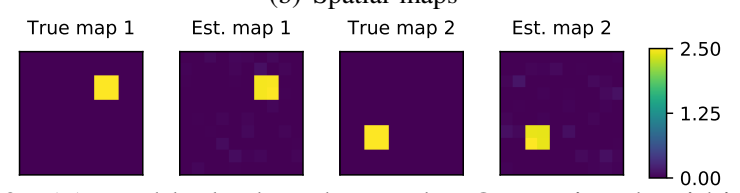

Fig. 2: (a) In black the observed BOLD signal within the associated activation region normalized by their $\ell_{\infty}$ norm, in blue the true temporal atoms, in orange the recovered temporal atoms. (b) The yellow-purple maps define the spatial ground truth and estimates. The standard deviation across voxels is encoded by transparency around mean curves.

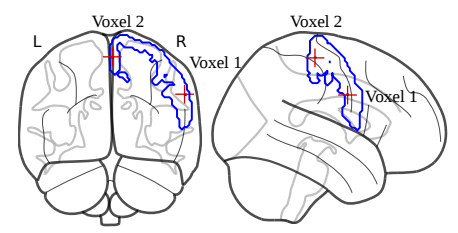

Fig. 3: Localization in the right Precentral Gyrus region of the two voxels (red crosses) chosen to illusrate the comparison between TA and LRD methods.

$1.0 \mathrm{~s}$ and a total scan duration of $1 \mathrm{~min} 40 \mathrm{~s}$ ( $T=100$ scans). We added a centered Gaussian noise such that the generated synthetic data has a signal-to-noise ratio of $1.0 \mathrm{~dB}$, defined by $S N R=10 \log _{10}\left(\frac{\left\|\sum_{k=1}^{K}\left(\boldsymbol{L} \boldsymbol{z}_{k}\right) \dot{*}\left(\boldsymbol{u}_{k} \boldsymbol{v}^{\top}\right)\right\|_{2}^{2}}{\|\boldsymbol{E}\|_{2}^{2}}\right)$.

Results. For this experiment, we chose $K=2$ and set the regularization parameter $\lambda$ to $0.4 \lambda_{\max }$, where $\lambda_{\max }$ is the minimal value for which $\mathbf{0}$ is solution of Eq. (3) and we fix the $\ell_{1}$-norm for each map to be equal to $\eta=10.0$. Fig. 2(a) displays the estimated temporal atoms $\left(\boldsymbol{L} \widehat{\boldsymbol{z}}_{1}, \boldsymbol{L} \widehat{\boldsymbol{z}}_{2}\right)$ in orange, along with the observed BOLD signals in black and the true signals in blue. Fig. 2(b) shows the corresponding spatial map estimates $\left(\widehat{\boldsymbol{u}}_{1}, \widehat{\boldsymbol{u}}_{2}\right)$ besides the ground truth. The temporal atom estimates recovered well the true signals. The block offsets are correctly temporally aligned but the rising and falling slopes of each block are not perfectly vertical. This indicates that their temporal derivative $\left(\widehat{\boldsymbol{z}}_{1}, \widehat{\boldsymbol{z}}_{2}\right)$ are not perfectly sparse. The spatial maps are adequately recovered as the activated regions are well localized and the map estimates are sparse.

\section{B. Results on real fMRI data}

Comparison to Total Activation approach. We qualitatively compare our BOLD low-rank decomposition (LRD) to the state-of-the-art method, called Total Activation (TA) [8]. In this approach, the authors propose to deconvolve the BOLD signal by minimizing a convex cost function that involved a 
sparse temporal constraint and a total variation spatial constraint. Their univariate approach allows to recover, a voxelspecific piecewise constant signal that models the neural activation signal as in our method. The main difference between the two methods is that our multivariate technique allows to recover a much easier-to-interpret decomposition of this neural activation signal. To reduce the computational cost of TA for this experiment, we only considered the temporal regularization.

HCP task fMRI data. Our validation was performed on the Human Connectom Project (HCP) dataset [18] which comprises fMRI recordings of participants performing different motor tasks. The tasks were adapted from the protocol developed in [19]. We chose this dataset as it presented both a good temporal and spatial resolution. A short time of repetition ( $\mathrm{TR}=720 \mathrm{~ms}$ ) was actually used to collect interleaved simultaneous multislice echo-planar images with a Multi-Band factor of 8 and a spatial resolution of $2 \times 2 \times 2 \mathrm{~mm}$. Each fMRI run lasted $3 \min 34 \mathrm{~s}$ in total during which $T=284$ scans were acquired. The fMRI data were already preprocessed using a classical pipeline including realignment, coregistration, spatial normalization and smoothing (5 $\mathrm{mm}$ isotropic). The experimental paradigm (EP) was divided in two sets of motor tasks, with 15 s fixation blocks at the beginning, in the middle and at the end of the recording. Each set was composed of 5 conditions, each modeled by a blocks of $12 \mathrm{~s}$, preceded by a $3 \mathrm{~s}$ cue indicating the task to be performed by the participant. The former corresponded to moving the tongue, tapping the left or right finger or squeezing the left or right toes. In what follows, we only consider one participant even though our results are reproducible across individuals.

Voxel selection. We aim to qualitatively compare the recovery of the neural activation signals for these two techniques (LRD and TA) in each voxel. Each fMRI run comprises a huge data set consisting of 230,314 voxels (time-courses). Thus, we only display results for a specific region the right Precentral Gyrus, corresponding to a subsample of 960 voxels in which we chose to display two voxels (see Fig. 3) illustrating the two methodologies.

Results. For this experiment, we chose $K=8$ as 8 experimental conditions were involved in the paradigm. As those approach are unsupervised models with no ground truth, we set the regularization parameter for LRD and TA by hand such that $\lambda_{T A}=0.02 \lambda_{\max }$ and $\lambda_{L R D}=0.07 \lambda_{\max }$. Last, we set the $\ell_{1}$-norm for each map of LRD to be equal to $\eta=10.0$. Fig. 4 illustrates the behavior of the LRD and TA deconvolution methods in these two voxels. In voxel-1, the low-rank neural activation signal (shown in blue) appears similar to the TA one. Both approaches mainly capture the same dynamics in the measured BOLD signal in this voxel. However, in voxel2, some high frequency components (short-duration activity) that are retrieved in the TA neural activation signal are not captured by our LRD method. This suggests that our model is less sensitive than TA in this voxel. As our temporal atoms are learned across voxels, this is a direct consequence of reducing (a) Voxel \#1
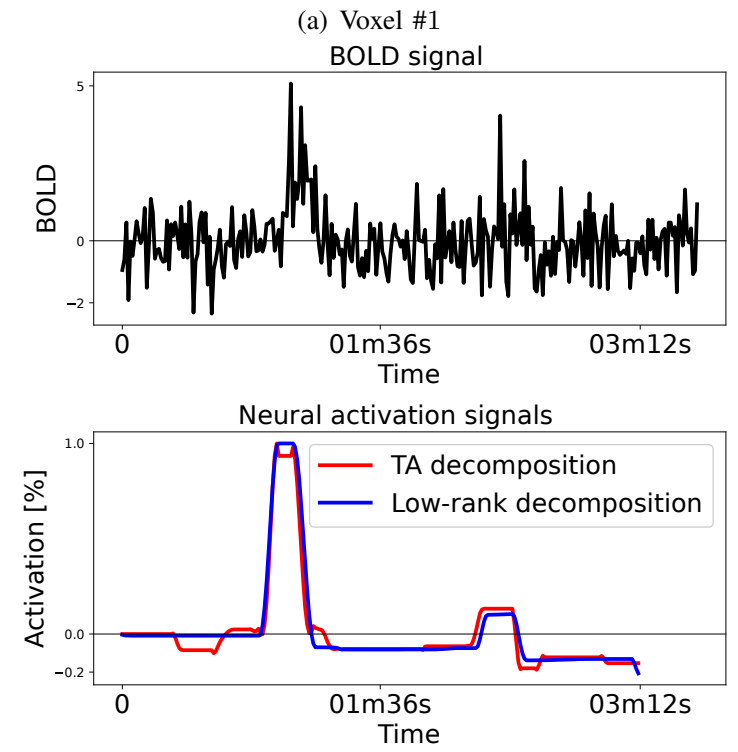

(b) Voxel \#2
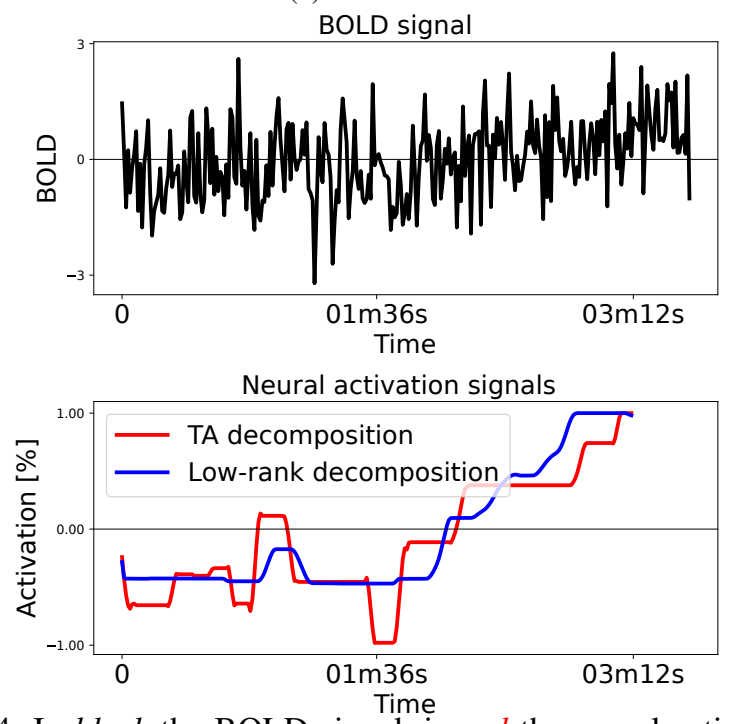

Fig. 4: In black the BOLD signal, in red the neural activation signal obtained using TA and in blue the neural activation signal obtained using our LRD approach.

the number of degrees of freedom in the temporal domain in LRD in contrast to TA. Fig. 5 and Fig. 6 depict respectively the temporal activities and the spatial maps associated to 2 of the 8 temporal atoms estimated with our LRD approach. Atom \#7 is mainly composed of two blocks locked to the offsets of the condition left hand and its spatial map is sparse, with a very well localized region of activation. This suggests that our model has learned the experimental condition that elicits brain activity in this region. In contrast, atom \#8 embodies a slightly rising slope between two constant periods, which illustrates its link to the low frequency fluctuations in the fMRI data. The second map displays smoother and wider activation areas in the right Precentral Gyrus, suggesting that this model is also capable of modeling trend effects, not related to the conditions. 
(a) Temporal atom \#7

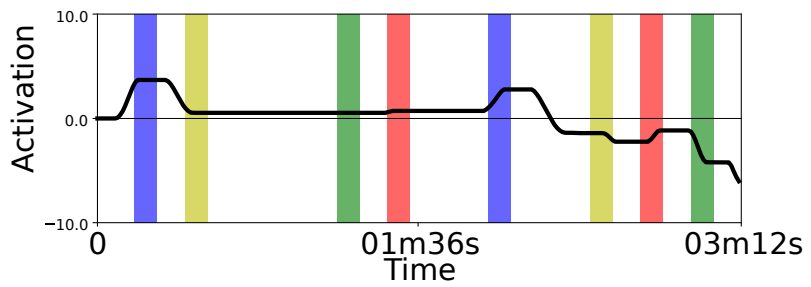

(b) Temporal atom \#8

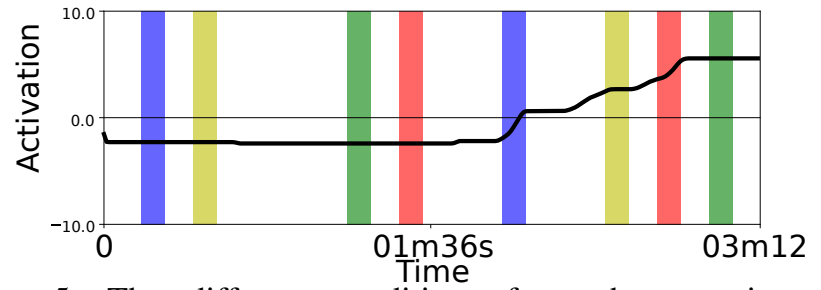

Fig. 5: The different conditions from the experimental paradigm are represented in background: blue for left hand, red for right hand, green for left foot and yellow for right foot. Each condition lasts $12 \mathrm{~s}$. On the foreground, the estimated temporal atom.

(a) Spatial map \#7

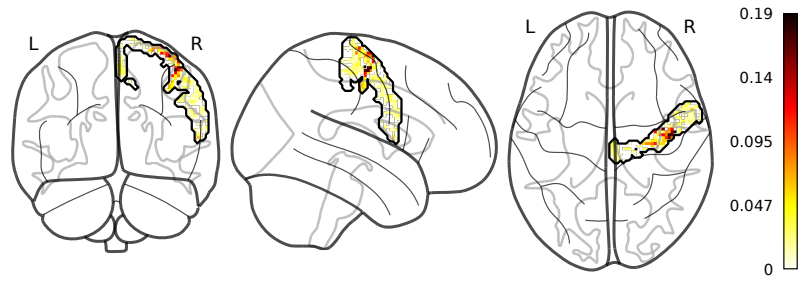

(b) Spatial map \#8

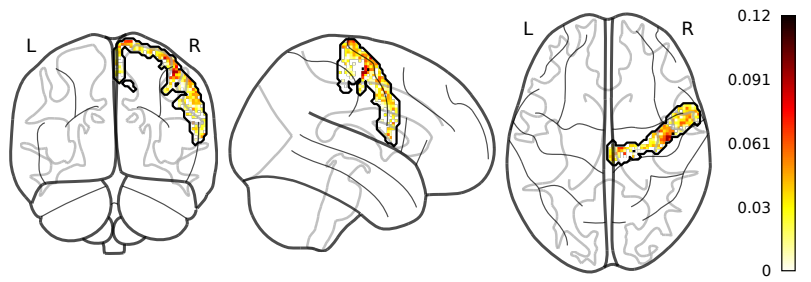

Fig. 6: The spatial maps associated with each atom. We have limited our analysis to the right Precentral Gyrus region (delimited in black). Values of estimated maps are color coded.

\section{CONClusions}

This paper presents a new low-rank decomposition modeling of the BOLD signal and a corresponding algorithm to perform both the deconvolution in time and the mapping in space. Although such low-rank modeling was already introduced in the dictionary learning literature [12], we adapted its formulation to fMRI data following ideas from the TA approach. In the validation on real fMRI data, we showed that our method provides a similar decomposition than TA. However, our multivariate model exhibited meaningful components that compose the BOLD signal along with their corresponding spatial maps. Nevertheless, the proposed algorithm remains too computationally demanding as the gradient steps involved all the voxels and time-frames. To reduce the computational cost in the future, we will investigate variable splitting approaches such as randomized block coordinate descents. This contribution opens new research avenues for inspecting functional connectivity networks that involve mostly the same atoms.

\section{ACKNOWLEDGMENT}

This work was supported by a CEA PhD scholarship, the UK Royal Academy of Engineering under the RF/201718/17128 grant and the SRPe PECRE 1718/15 Award.

\section{REFERENCES}

[1] S. Ogawa, D. W. Tank, R. Menon, J. M. Ellerman, S. G. Kim, H. Merkle, and K. Ugurbil, "Intrinsic signal changes accompanying sensory stimulation: functional brain mapping with magnetic resonance imaging," in Proceedings of the National Academy of Sciences, vol. 89, 1992, pp. 5951-5955.

[2] P. A. Bandettini, A. Jesmanowicz, E. C. Wong, and J. S. Hyde, "Processing strategies for time-course data sets in functional MRI of the human brain," Magnetic Resonance in Medicine, vol. 30, pp. 161-173, 1993.

[3] G. M. Boynton, S. A. Engel, G. H. Glover, and D. J. Heeger, "Linear systems anlaysis of functional magnetic resonance imaging in human V1," Journal of Neuroscience, vol. 16, no. 13, pp. 4207-4221, 1996.

[4] K. J. Friston, A. P. Holmes, C. J. Price, C. Buchel, and K. J. Worsley, "Multisubject fMRI studies and conjunction analyses," NeuroImage, vol. 10, pp. 385-396, 1999.

[5] R. Viviani, G. Grön, and M. Spitzer, "Functional principal component analysis of fMRI data." in Human Brain Mapping, vol. 24, 2005, pp. 109-29.

[6] C. Beckmann and S. Smith, "Probabilistic independent component analysis for functional magnetic resonance imaging." in IEEE Transactions on Medical Imaging, vol. 23, 2004, pp. 137-152.

[7] G. Varoquaux, S. Sadaghiani, P. Pinel, A. Kleinschmidt, J. B. Poline, and B. Thirion, "A group model for stable multi-subject ICA on fMRI datasets," NeuroImage, vol. 51, pp. 288-299, 2010.

[8] F. I. Karahanoglu, C. Caballero-Gaudes, F. Lazeyras, and D. Van De Ville, "Total activation: fMRI deconvolution through spatio-temporal regularization," NeuroImage, vol. 73, pp. 122-134, 2013.

[9] C. Caballero-Gaudes, F. I. Karahanoglu, F. Lazeyras, and D. Van De Ville, "Structured sparse deconvolution for paradigm free mapping of functional MRI data," in International Symposium on Biomedical Imaging, vol. 9, 2012, pp. 322-325.

[10] H. Cherkaoui, T. Moreau, A. Halimi, and P. Ciuciu, "Sparsity-based blind deconvolution of neural activation signal in fMRI," in International Conference on Acoustics, Speech and Signal Processing, 2019.

[11] B. A. Olshausen and D. J. Field, "Sparse coding with an incomplete basis set: a strategy employed by V1," Vision Research, vol. 37, no. 23, pp. 3311-3325, 1997.

[12] T. Dupre La Tour, T. Moreau, M. Jas, and M. Gramfort, "Multivariate convolutional sparse coding for electromagnetic brain signals," Advances in Neural Information Processing Systems, vol. 31, 2018.

[13] P. Ciuciu, J. B. Poline, G. Marrelec, J. Idier, C. Pallier, and H. Benali, "Unsupervised robust non-parametric estimation of the hemodynamic response function for any fMRI experiment," IEEE Transactions on Medical Imaging, vol. 22, pp. 1235-1251, 2003.

[14] M. A. Lindquist, J. Meng Loh, L. Atlas, and T. Wager, "Modeling the hemodynamic response function in fMRI: efficiency, bias and mismodeling," NeuroImage, vol. 45, pp. S187-S198, 2009.

[15] A. Beck and M. Teboulle, "A fast iterative shrinkage-thresholding algorithm for linear inverse problems," SIAM Journal on Imaging Sciences, vol. 2, pp. 183-202, 2009.

[16] L. Armijo, "Minimization of functions having lipschitz continuous first partial derivatives," MathSciNet, 1966.

[17] L. Condat, "Fast projection onto the simplex and the 11 ball," Mathematical Programming Series A, vol. 158, pp. 575-585, 2016.

[18] D. C. Van Essen, S. M. Smith, D. M. Barch, T. E. J. Behrens, E. Yacoub, and K. Ugurbil, "The WU-minn human connectome project: An overview," NeuroImage, vol. 80, pp. 62-79, 2013.

[19] B. T. T. Yeo and et al, "The organization of the human cerebral cortex estimated by intrinsic functional connectivity," Journal of Neurophysiology, vol. 106, pp. 1125-1165, 2011. 